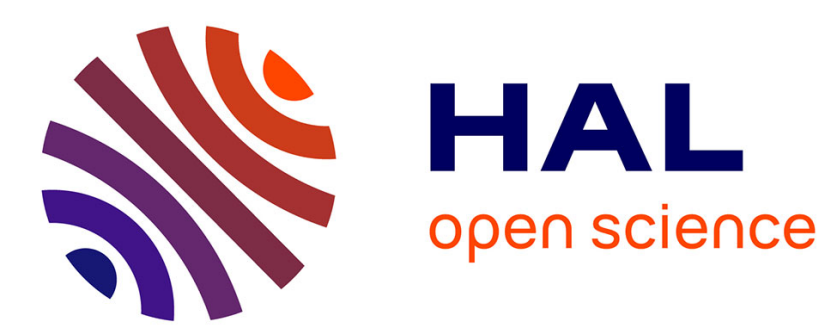

\title{
DURATION OF COLLISIONS IN SEMICONDUCTORS
}

J.-P. Nougier, J. Vaissière, D. Gasquet

\section{To cite this version:}

J.-P. Nougier, J. Vaissière, D. Gasquet. DURATION OF COLLISIONS IN SEMICONDUCTORS. Journal de Physique Colloques, 1981, 42 (C7), pp.C7-283-C7-292. 10.1051/jphyscol:1981734 . jpa00221671

\section{HAL Id: jpa-00221671 https://hal.science/jpa-00221671}

Submitted on 1 Jan 1981

HAL is a multi-disciplinary open access archive for the deposit and dissemination of scientific research documents, whether they are published or not. The documents may come from teaching and research institutions in France or abroad, or from public or private research centers.
L'archive ouverte pluridisciplinaire HAL, est destinée au dépôt et à la diffusion de documents scientifiques de niveau recherche, publiés ou non, émanant des établissements d'enseignement et de recherche français ou étrangers, des laboratoires publics ou privés. 


\title{
DURATION OF COLLISIONS IN SEMICONDUCTORS
}

\author{
J.P. Nougier, J.C. Vaissiěre and D. Gasquet \\ Université des Sciences et Techniques du Lronguedoc, Centre d'Etudes d'EZectro- \\ nique des Solides, Laboratoire associé au C.N.R.S., LA 21, Greco Microondes et \\ G.CIS., 34060 Montpelzier Cedex, Fronce
}

\begin{abstract}
Rêsumé.- Une des hypothèses de validité de 1 'équation de Boltzmann est que Tes collisions sont instantanées. Nous montrons dans cet article que, pour les interactions usuelles dans les semiconducteurs, la durée de collision peut être estimée $\mathrm{a} \simeq 5 \times 10^{-13} \mathrm{sec}$ et $\mathrm{n}^{\prime}$ est donc pas négligeable devant $1 \mathrm{a}$ durẻe de libre parcours moyen.

Abstract.- One of the basic hypothesis involyed in the Boltzmann equation is that the collisions are instantaneous. In this paper it is shown that, for usual scattering processes in semiconductors, the collision duration can be estimated to be $\simeq 5 \times 10^{-13} \mathrm{sec}$, which is therefore not at all negligible compared with the mean free flight duration.
\end{abstract}

\section{INTRODUCTION}

Transport coefficients in semiconductors are defined as averaged values of functions of the wave vector $\vec{k}$, over the distribution function $f(\vec{k}, \vec{r}, t)$ which, in an electric field $\vec{E}(\vec{r})$, is defined as a solution of the classical Boltzmann equation :

$$
\frac{\partial f(\vec{k}, \vec{r}, t)}{\partial t}+\frac{e \vec{E}}{\hbar} \cdot \vec{\nabla}_{k} f(\vec{k}, \vec{r}, t)+\vec{v} \vec{\nabla}_{r} f(\vec{k}, \vec{r}, t)=\widehat{c} f(\vec{k}, \vec{r}, t)
$$

$\hbar=h / 2 \pi, h$ and e are the Planck's constant and the charge of a carrier, $\hbar \vec{k}$ is the quasi momentum and $\hat{c}$ is the collision operator. In the classical formulation, $\hat{c}$ is defined as:

$$
\begin{aligned}
& \widehat{c} f(\vec{k}, \vec{r}, t)=\sum_{\vec{k}^{\prime}} P\left(\vec{k}^{\prime}, \vec{k}\right) f\left(\vec{k}^{\prime}, \vec{r}, t\right)[1-f(\vec{k}, \vec{r}, t)] d^{3} k^{\prime} \\
&-\sum_{\vec{k}^{\prime}} P\left(\vec{k}^{\prime}, \vec{k}^{\prime}\right) f(\vec{k}, \vec{r}, t)\left[1-f\left(\vec{k}^{\prime}, \vec{r}, t\right)\right] d^{3} k^{\prime}
\end{aligned}
$$


and for usual doping $f \ll<. P\left(\vec{k}, \vec{k}^{\prime}\right)$ is the transition rate from the state $\vec{k}$ to the state $\vec{k}^{\prime}$. Among the approximations made for getting eq. (2), is the assumption that the collisions are instantaneous, which means that $P\left(\vec{k}, \vec{k}^{\prime}\right)$ does not depend on time, which has two consequences : (i) $P\left(\vec{k}, \vec{k}^{\prime}\right)$ does not depend on the electric field, (ii) after a collision, a carrier has lost the memory of its initial state, which means that the process is Markovian. Thus the memory time of a given carrier is mainly related to the time between two successive collisions, called the relaxation time, or the free flight duration, which is well known to lie in the range $10^{-12}$ - $10^{-14} \mathrm{sec}$. When the assumption of the instantaneous collisions fails, the classical Boltzmann eq. (1) must be replaced by a "retarded" equation [1] [2].

It is then very important to get an idea of the order of magnitude of the average collision duration, which needs first to define it. A possible definition, in connection with our problem, could be the following : the collision duration is the time needed for the distribution function to loose the memory of its earlier states. This definition, which is similar to that of a correlation time, would indeed give the time below which the classical Boltzmann equation should be replaced by a retarded equation. Unfortunately, we are up to now unable to evaluate this time. Because of the probabilistic nature of the quantum mechamical equations, we can but estimate it as being the time during which the carrier is under the influence of the scattering center. As a consequence, the classical Boltzmann equation will be valid when the duration of a collision (just defined above), is short compared with the free flight duration, that is with the average time between two collisions.

This can be physically illustrated by making a Monte Carlo simulation, which was proved $[3][4]$ to be a solution of the classical Boltzmann equation. In such a simulation, a free flight duration is determined using a random number, which allows one to know the state of the carrier at the end of the free flight, which means its initial state $\vec{k}$ at the beginning of the next collision. After having selected the collision mechanism using an other random number, three more random numbers allow one to determine the final state $\vec{k}^{\prime}$ after the collision. In fact only two random numbers are needed since the find energy $\varepsilon^{\prime}$ is determined through the conservation law $\varepsilon^{\prime}=\varepsilon \pm h \omega$. Thus the final state and the final energy are simultaneously determined at the end of the free flight. The transition does not depend on the electric field since the carrier has not time to be accelerated during the collision, as it is during the free flight.

The purpose of this paper is not to evaluate the correct collision duration $\tau_{c}$ involved in ref.| $1 \| 2 \mid$, which is much difficult since $\tau_{c}$ depends both on the scattering mechanisms and on the distribution function itself. Rather, we shall point out the inconsistency of the usual Boltzmann equation, by showing that the collision duration $\tau_{c}$ involved in it, deduced from well known formulas, is not at 
all negligible compared with the free flight duration. As a consequence the lecturer will not find in this paper any guidance for deducing the correct value of $\tau_{c}$. Therefore one might be doubtful as concerning the usefulness of such a paper. Indeed due to the numerous discussions arising about the necessity of using retarded transport equations, we feel necessary to clarify some misunderstandings about usual concepts of collisions and, mainly, to give some numerical values, which has never been done previously, so that people keep in mind the orders of magnitudes of the phenomena involved.

We shall first investigate classical motions in a well of constant potential (section 2) and in a screened coulomb potential (section 3), then quantum scattering by phonons (section 4 ).

\section{CLASSICAL SCATTERING BY A WELL OF CONSTANT POTENTIAL}

An incident carrier is scattered by a potential $U(\vec{r})$ :

$U(\vec{r})=-U_{0}$ for $r \leqslant R$ and $U(\vec{r})=0$ for $r>R$

Let $b$ and $v_{\infty}$ be the parameters defining the incident particle of mass $m$. The conservation of the energy gives :

$$
\frac{1}{2} m v_{\infty}^{2}=\frac{1}{2} m v^{2}-u_{0}
$$

and the incident and refraction angles $\alpha$ and $\beta$ (see fig. 1) are related through : $\sin \alpha / \sin \beta=\left(1+2 U_{0} / \mathrm{mv}_{\infty}{ }^{2}\right)^{1 / 2}$

Figure 1 : Classical trajectory of a particle scattered by a we 11 of constant attractive potential.

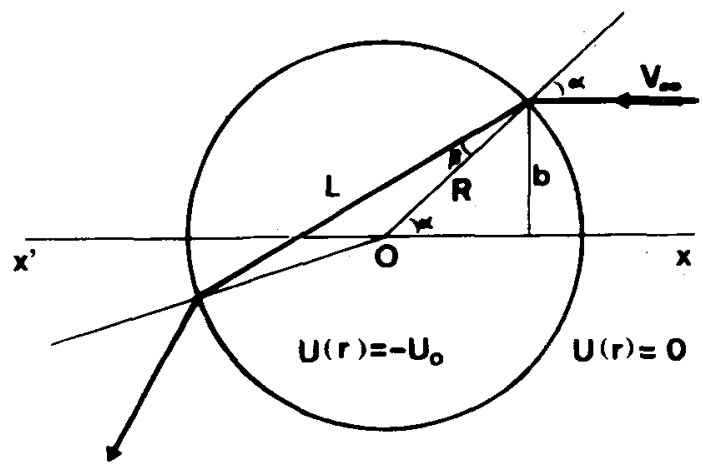


The length of the path $L$ in the well (see Fig. 1) is $L=2 R \cos \beta$, the duration of the collision is then $t\left(b, v_{\infty}\right)=L / v$ that is :

$$
t\left(b, v_{\infty}\right)=2\left[R^{2}\left(1+2 u_{0} / m v_{\infty}^{2}\right)-b^{2}\right]^{1 / 2} v_{\infty}^{-1}\left(1+2 u_{0} / m v_{\infty}^{2}\right)^{-1}
$$

The $d n$ particles in the range $[b, b+d b]$ is $d n \propto 2 \pi b d b$, if one supposes a uniform density. The average collision time $\boldsymbol{x}(\mathrm{b})$ is then :

$$
\tau\left(v_{\infty}\right)=\int_{b=0}^{R} t\left(b, v_{\infty}\right) d n / \int_{b=0}^{R} d n=\int_{0}^{R} t\left(b, v_{\infty}\right) b d b / \int_{0}^{R} b d b
$$

that is:

$$
\tau\left(v_{\infty}\right)=\frac{4 R}{3 v_{\infty}}\left[\left(1+2 u_{0} / \mathrm{mv}_{\infty}^{2}\right)^{3 / 2}-\left(2 u_{0} / \mathrm{mv}_{\infty}^{2}\right)^{3 / 2}\right]\left(1+2 \mathrm{u}_{0} / \mathrm{mv}_{\infty}^{2}\right)^{-1}
$$

At the present stage, several remarks must be made :

(i) The beginning $t_{i}$ and the end $t_{f}$ of a collision are perfectly determined. The collision is elastic, thus the initial and final energies are equal, but the final state (or velocity) is different from the initial one since the particle is deflected. (ii) During the collision, the energy departs from its initial value.

(iii) The motion is classical, which means that, when the initial state is known, the final state is perfectly determined.

This example shows that there are at least two ways for defining the duration of the collision $t\left(b, v_{\infty}\right)$ :

a) the time during which the energy departs from its initial and final values

( $\varepsilon=\varepsilon_{j}$ and $\varepsilon=\varepsilon_{f}$ ).

b) the time during which the velocity (= the state) departs from its initial and

final values, that is the time during which $0<\left(\overrightarrow{0} x^{\prime}, \vec{v}_{\infty}\right)<\chi$ where $\chi=2(\alpha-\beta)$

is the deflection angle.

In this example, these two times are identical.

Now $\tau\left(v_{\infty}\right)$ can be computed, we used for this purpose the parameters corresponding to $n-S i$ at $300 \mathrm{~K}$ with $n=N_{D}=10^{15} \mathrm{~cm}^{-3}$. $R$ was set equal to the average distance between two impurity atoms $R=5 \times 10^{-8} \mathrm{~m}$. $U_{0}$ was set equal to the average value of a screened Coulomb potential in the sphere of radius $R$ : 
Figure 2: Average collision duration versus the initial velocity for classical motions of a particle of mass $0.26 \mathrm{~m}_{0}$, in a well of constant potential (CP) and in a screened Coulomb potential (SCP). Parameters used : $C P: U(r)=-2.87 \mathrm{meV}$ for $r \leqslant 5 \times 10^{-8} \mathrm{~m}, U(r)=0$ for $r>5 \times 10^{-8} \mathrm{~m}$.

SCP : parameters of silicon at $300 \mathrm{~K}, \mathrm{~N}_{\mathrm{D}}=1015 \mathrm{~cm}^{-3}$.

$\rightarrow C P$; SCP, collision beginning when the velocity vector deviates of 0.01 rad from its initial value $(-)$, when the kinetic energy departs of $10^{-6}$ from its initial value $(-)$, when $r=R(-)$, and $r=a \quad(\rightarrow-)$

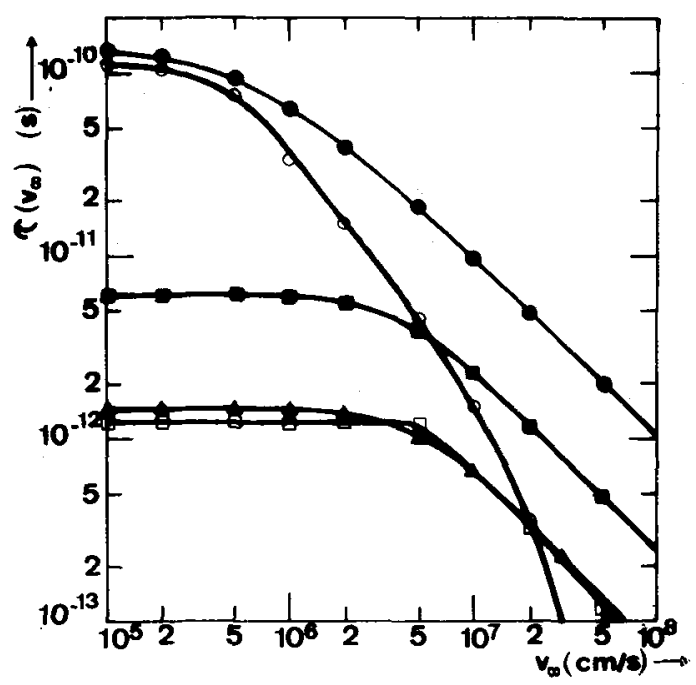

$$
-U_{0}=\int_{0}^{R} U(\vec{r}) r^{2} d r / \int_{0}^{R} r^{2} d r
$$

$U(\vec{r})$ is given in the next section. This leads to $U_{0}=2.87 \mathrm{meV}$.

Figure 2 shows the variation of $\tau\left(v_{\infty}\right)$. It follows from eq. (4) that $\tau\left(v_{\infty} \rightarrow 0\right)=2 R \sqrt{m / 2 U_{0}}$ and $\tau\left(v_{\infty} \rightarrow \infty\right) \simeq 4 R / 3 v_{\infty} \rightarrow 0$. Figure 2 shows that, for the usual initial velocities of the carriers $v_{\infty} \leqslant 10^{7} \mathrm{~cm} / \mathrm{s}, \tau \simeq 1 \mathrm{ps}$, which means that $\tau$ is of the order of magnitude of the free flight duration.

\section{CLASSICAL SCATTERING BY A SCREENED COULOMB POTENTIAL}

$$
\begin{aligned}
& \text { Now } \\
& U(r)=-\frac{A}{r} \exp (-r / a) \\
& A=e^{2} / 4 \pi \zeta \text { and } a=\left(\zeta k_{B} T / n e^{2}\right)^{1 / 2}
\end{aligned}
$$

where $\zeta$ is the dielectric constant, $k_{B}$ the Boltzmann constant. Let $(r, \theta)$ be the polar coordinates, of the incoming particle of initial energy $\varepsilon=\frac{1}{2} \mathrm{mv}_{\infty}^{2}$ and angular momentum $J=m v_{\infty}$ b. Given $(r, \theta)$, one has :

$$
\left\{\begin{array}{l}
(d r / d t)^{2}=2 m^{-1}[\varepsilon-U(r)]-\left(J^{2} / m^{2} r^{2}\right) \\
d \theta / d t=J / m r^{2}
\end{array}\right.
$$

These eqs allow one to get $r(t+\Delta t)=r(t)+\Delta t d r / d t$ and $\theta(t+\Delta t)=\theta(t)+\Delta t d \theta / d t$. The trajectory can then be computed. 
Some difficulties arise, for defining the collision time, with respect to the previous example, related to the fact that the potential well is not bounded. Thus, strictly speaking, the collision time is infinite. In practice, when the particle is far from the diffusing center, the potential is so low that the energy and the velocity are almost unchanged. As a consequence we defined the collision duration as $t\left(b, v_{\infty}\right)=2\left(t_{m}-t_{j}\right)$ where $t_{m}$ is the time when $r$ is minimum, that is $(d r / d t)^{2}=0$. For $t_{i}$ several somewhat arbitrary definitions can be used :

a) $t_{j}=t_{i v}$ such as the velocity deviates significantly from its initial direction, namely $\left\langle\overrightarrow{0} x^{\prime}, \vec{v}\right)=0.01$ rad. This gives $\tau_{v}\left(b, v_{\infty}\right)=2\left(t_{m}-t_{i v}\right)$.

b) $t_{j}=t_{j \varepsilon}$ such as the kinetic energy departs significantly from its initial value, namely : $-U\left[r\left(t_{i \varepsilon}\right)\right] / \varepsilon=10^{-6}$. This gives $t_{\varepsilon}\left(b, v_{\infty}\right)=2\left(t_{m}-t_{i \varepsilon}\right)$.

c) $t_{i}=t_{i R}$ such as $r\left[t_{i R}\right]=R$ where $R$ is the half mean distance of two impurities : $R=5 \times 10^{-8} \mathrm{~m}$ for $N_{D}=10^{15} \mathrm{~cm}^{-3}$. This gives $t_{R}\left(b, v_{\infty}\right)$.

d) $t_{i}=t_{i a}$ such as $r\left[t_{i a}\right]=a$. This gives $t_{a}\left(b, v_{\infty}\right)$.

Once the $t\left(b, v_{\infty}\right)$ have been computed, one gets the average collision duration $\tau\left(v_{\infty}\right)$ through eq. (4). Using the parameters of $n-S i$ at $300 \mathrm{~K}, N_{D}=10^{15} \mathrm{~cm}^{-3}$, one gets $A=1.97 \times 10^{-29} \mathrm{~m}$ and $a=1.29 \times 10^{-7} \mathrm{~m}$. Figure 2 shows $\tau_{v}\left(v_{\infty}\right), \tau_{\varepsilon}\left(v_{\infty}\right)$, $\tau_{R}\left(v_{\infty}\right)$ and $\tau_{a}\left(v_{\infty}\right)$ versus $v_{\infty}$. Of course, fig. 2 shows that the different definitions lead to different collision durations. However, in the range $v_{\infty}=10^{5}$ to $10^{7} \mathrm{~cm} / \mathrm{s}$, the collision durations exceeds $5 \times 10^{-13} \mathrm{sec}$.

4. QUANTUM SCATTERING

In quantum scattering, the final state is not determined once the initial state is known. If a carrier at time $t=0$ undergoes a collision with, say, a phonon of energy $\hbar \omega$, the probability $\mathcal{P}_{t}\left(\vec{k}, \vec{k}^{\prime}\right)$ of a transition, between its state $\vec{k}$ of energy $\varepsilon$ at time $t=0$, and the state $\vec{k}^{\prime}$ of energy $\varepsilon^{\prime}$ at time $t$, is given by :

$$
\mathcal{S}_{t}\left(\vec{k}, \vec{k}^{\prime}\right)=\left|V_{k k^{\prime}}\right|^{2} 4\left[\sin ^{2} \frac{\left(\varepsilon-\varepsilon^{\prime} \pm \hbar \omega\right) t}{2 \hbar}\right] /\left(\varepsilon-\varepsilon^{\prime} \pm h \omega\right)^{2}
$$

where the plus and minus signs correspond to absorption and emission. For elastic scattering, $\hbar \omega=0$. Setting

$$
\alpha=\left(\varepsilon^{\prime}-\varepsilon_{f}\right) / 2 \hbar \text { and } \varepsilon_{f}=\varepsilon \pm \hbar \omega
$$

eq. (6) writes :

$$
\mathcal{P}_{t}\left(\vec{k}, \vec{k}^{\prime}\right)=\frac{\pi\left|v_{k k^{\prime}}\right|^{2}}{\hbar^{2}}+\frac{\sin ^{2} \alpha t}{\pi \alpha^{2} t}
$$

Usually one sets $\sin ^{2} \alpha t /\left(\pi \alpha^{2} t\right)=\delta(\alpha)$, which gives $\varepsilon^{\prime}=\varepsilon_{f}$ (energy conservation): this is true when $t \rightarrow \infty$, in practice when $|\alpha t| \gg>\pi$. 
In this approximation, the transition rate becomes constant :

$d S_{t}\left(\vec{k}, \vec{k}^{\prime}\right) / d t=\pi\left|V_{k k^{\prime}}\right|^{2} \delta(\alpha) / \hbar^{2}=P\left(\vec{k}, \vec{k}^{\prime}\right)$, and one gets the classical Boltzmann equation.

It is often assumed, when writing that the transition between the state $\vec{k}$ at time $t=0$ and the state $\vec{k}^{\prime}$ at time $t$ is $\vec{S}_{t}\left(\vec{k}, \vec{k}^{\prime}\right)$, that this transition takes place suddenly at time $t$, within $d t$, nothing being occured between 0 and $t-d t$ : if this description were valid, the collision could actually be instantaneous (the collision duration being $d t$ ), although occuring within the time delay $t$. However this picture is erroneous, and the transition occurs gradually during the whole time duration $t$. The reason is that, if the transition occured suddenly, $S_{t}\left(\vec{k}^{,}, \vec{k}^{\prime}\right)$ would exhibit sudden changes, since one would get $\mathcal{S}_{t-d t}\left(\vec{k}, \vec{k}^{\prime}\right)=0$ and $\vec{S}_{t}\left(\vec{k}^{\prime}, \vec{k}^{\prime}\right) \neq 0$ : hence $d S_{t}\left(\vec{k}, \vec{k}^{\prime}\right) / d t$ would show discontinuities. Indeed the carrier initially in the state $\vec{k}$ (labelled $\mid \vec{k}>$ in quantum mechanics) at time $t=0$, is at time $t$ in the state $U(t, 0) \mid \vec{k}>$, where $U(t, 0)$ is the evolution operator solution of the equation $|6|$ :

$$
i \hbar \partial U(t, 0) / \partial t=H(t) U(t, 0)
$$

where $H(t)$ is the total hamiltonian including the scattering potential $V(t)$ (as well as the external field). The probability to find this carrier in the state $\left|\vec{k}^{\prime}\right\rangle$ at time $t$ is then :

$$
\Gamma_{t}\left(\vec{k}, \vec{k}^{\prime}\right)=\left|\left\langle\vec{k}^{\prime}|u(t, 0)| \vec{k}\right\rangle\right|^{2}
$$

As can be seen from eq. (9), the variation of $U(t, 0)$ is quite gradual, and so is the variation of $\vec{S}_{t}\left(\vec{k}, \vec{k}^{\prime}\right)$. Thus the transition between the state $\vec{k}$ at time 0 and the state $\vec{k}^{\prime}$ at time $t$ actually lasts during all the time $t$. of course, within the same time $t$, the transition may as well occur between the states $\vec{k}$ and $\vec{k}^{\prime \prime}$, with the probability $\mathcal{S}_{t}\left(\vec{k}, \vec{k}^{\prime \prime}\right)$ : if it occurs, this transition is also gradual.

The important above comments lead to the conclusion that the time $t$ is, more or less, related to the duration of the transition between the states $\vec{k}$ and $\vec{k}^{\prime}$, that is to the duration of the collision. In order to define this more precisely, we have plotted, on figure $3, \widehat{S}_{t}\left(\varepsilon^{\prime}-\varepsilon_{f}\right) / \widehat{S}_{t}(0)=(\sin \alpha t / \alpha t)^{2}$ as a function of $\varepsilon^{\prime}-\varepsilon_{f}$ for various values of $t$ between $10^{-15} \mathrm{sec}$ and $5 \times 10^{-13} \mathrm{sec}$. $\mathcal{P}_{t}\left(\varepsilon^{\prime}-\varepsilon_{f}\right)$ is then obtained by multiplying this quantity by a factor proportional to $t^{2}$. We shall define the duration of the collision $\tau(\vec{k})$ as the time needed for the carrier to reach its final energy, $i . e$. the time such that $\varepsilon^{\prime}-\varepsilon_{f} \approx 0$. As was already noticed, one might have defined it as being the time $\tau^{\prime}(\vec{k})$ needed to reach the final state, but eq. (6) does not give any information about that. However, because of the dispersion law $\varepsilon(\vec{k})$, once the final state has been reached, the final energy is $\varepsilon_{f}$; on the contrary, since many states have the same energy, the final energy $\varepsilon_{f}$ can be reached although the carrier is not still in its final state. 
Now the time needed for $\varepsilon^{\prime}-\varepsilon_{f}=0$ is infinite. In practice, we must define the collision time as being the time such that $\varepsilon^{\prime}-\varepsilon_{f}$ is located around zero with a good enough accuracy. For example it can be assumed that emission or absorption of an optical phonon of typically $40 \mathrm{meV}$ is achieved when the energy $\varepsilon^{\prime}$ departs from $\varepsilon_{f}$ of an amount of about $10 \%$ of the phonon energy, that is $\varepsilon^{\prime}-\varepsilon_{f} \leqslant 4 \mathrm{meV}$. Fig. 4 shows that it takes $5 \times 10^{-13} \mathrm{sec}$ for the probability, that $\varepsilon^{\prime}-\varepsilon_{f} \leqslant 4$ meV, to be high enough compared with the probability that $\varepsilon^{\prime}-\varepsilon_{f}>4 \mathrm{meV}$. For example the curve $t=10^{-13} \mathrm{sec}$ on Fig. 4 shows that the final energy is located within $\pm 40 \mathrm{meV}$ around $\varepsilon_{f}$. Of course the value $4 \mathrm{meV}$ has been taken arbitrary, but it gives a fairly good order of magnitude. For elastic scattering (acoustical phonons or impurities), one might have decided that the collision is achieved when the energy $\varepsilon^{\prime}$ departs from $\varepsilon_{f}=\varepsilon$ of an amount of $0.1 \mathrm{kT}$, that is $2.5 \mathrm{meV}$ at $300 \mathrm{~K}$, Teading to a time slightly larger than $5 \times 10^{-13} \mathrm{sec}$. Note that the collision time defined through the final state rather than through the final energy, as was discussedabove, is still larger.

This section clearly shows that $5 \times 10^{-13} \mathrm{sec}$ gives an order of magnitude of the duration of a collision, which is therefore not at all negligible compared with the free flight duration.

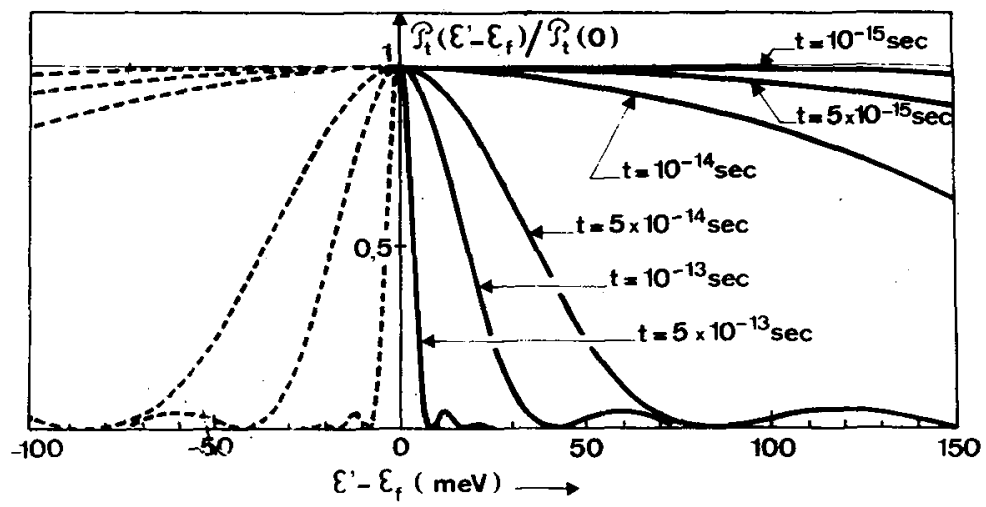

Figure 3 : Normalized transition probability versus the energy $\varepsilon^{\prime}-\varepsilon_{f}$ of the scattered particle, at various instants. $\varepsilon_{f}=\varepsilon \pm \hbar \omega$ is the final energy reached after an infinite time. 


\section{CONCLUSION}

The evaluation of the duration of a collision is a fundamental problem for deciding whether one should use the classical Boltzmann equation or a retarded equation. However, as was shown in this paper, this problem is very hard to solve, because many definitions of the collision time can be used, since several parameters vary during a collision. Once a parameter has been choosen, the magnitude of its variation still remains arbitrary : this was clearly evidenced even on examples in classical mechanics.

A very simple evaluation of the collision duration can be performed using the laws of classical mechanics for impurity scattering : the radius of influence of an impurity is $\simeq 10^{-7} \mathrm{~m}$, so that a carrier having a drift velocity in the range $10^{5}$ to $10^{7} \mathrm{~cm} / \mathrm{s}$ will remain $10^{-10}$ to $10^{-12} \mathrm{sec}$ under its influence : this is in agreement with the results shown fig. 2 .

For a deeper insight, we must use a quantum formalism of the transition probabilities : according to the discussion of section 4 , illustrated by Fig. 3 , we got an estimate of the collision duration of $\simeq 5 \times 10^{-13} \mathrm{sec}$. This is of the order of magnitude of the free flight duration. We therefore conclude that the classical Boltzmann equation, in which the collision duration is neglected, is not very well appropriate for describing transport in semiconductors.

It must be taken in mind that the time discussed above is only an estimate of the usual, approximate, expression of the collision duration : it is not an estimate of the exact collision time $\tau_{c}$, which depends on the strength of the interaction as well as on the external driving force. However it likely gives the order of magnitude of the phenomenon, which has not yet been performed till now, and anyway shows the inconsistency of the hypothesis leading to the usual Boltzmann equation for semiconductors.

The question is then to know whether this can significantly or not modify the numerous results, in particular of transient regimes, accumulated during the last decade by solving the classical Boltzmann equation. Indeed one would think that actual relaxation mechanisms are less efficient than given by the instantaneous collision approximation, since emission of optical or intervalley phonons, which are the main relaxation mechanisms, require much more time than given by the classical theory. In fact one must keep in mind that, using new equations, new coupling constants (deformation potentials, etc...) should be used, so as to fit the new theoretical results with the known experimental data of static transport coefficients. As a consequence, the transient regimes themselves probably will not be much modified : indeed it can be shown [5] that transient behaviour can be quite correctly described using balanced equations involving the static characteristics, independently 
from any hypothesis concerning the scattering mechanisms or the distribution function. However all these predictions are but qualitative and should be confirmed by computations performed on concrete examples.

\section{REFERENCES}

[1] J.R. BARKER, Solid St. Electron. 21, 267 (1978)

[2] D.K. FERRY and J.R. BARKER; Solid St. Commun. 30, 301 (1979)

[3] P.A. LEBWOHL, J. App]. Phys. 44, 1744 (1973)

[4] P.J. PRICE, Proc. Int. Conf. Phys. Semicond. Moscow, Vol.2 (Leningrad Nauka) p. 753 (1968).

[5] L. REGGIANI, J.C. VAISSIERE, J.P. NOUGIER, D. GASQUET, Transient regimes of hot carriers in p-type silicon, Proc. 3rd. Int. Conf. on Hot Carriers in Semiconductors, Montpellier (France), 7-10 July 1981, J. de Physique Colloques, $\mathrm{C} 7$.

[6] A. MESSIAH, Mécanique Quantique, Dunod ed., 1964, Chap. 17. 